\title{
Apoptosis in Transitional Cell Carcinoma of Bladder and its Relation to Proliferation and Expression of P53 and Bcl-2
}

\author{
Zahra AMIRGHOFRAN, ${ }^{1,2}$ Ahmad MONABATI, ${ }^{3}$ Abdolaziz KHEZRI, ${ }^{4}$ Zahra MALEK-HOSSEINI $^{1}$ \\ Departments of ${ }^{1}$ Immunology, ${ }^{3}$ Pathology and ${ }^{4}$ Urology, Medical School, ${ }^{2}$ Medicinal and Natural Product \\ Chemistry Research Center, Shiraz University of Medical Sciences, Shiraz, Iran
}

Transitional cell carcinoma of bladder (TCC) is a relatively common cancer among men. Tumor progression is associated with expression or modulation of several gene products that control apoptosis and proliferation. Apoptosis is a negative growth regulatory mechanism in tumors. The aim of this study is to examine apoptosis and related regulatory molecular markers in a group of patients with TCC. Paraffinembedded tissues from 49 patients with TCC were examined for the expression of bcl-2, p53 and Ki-67 by immunohistochemistry. Apoptosis was detected by TUNEL method. Correlation between apoptotic index (AI), proliferation index (PI) and bcl-2 and p53 expression with each other and with pathological grade was determined. Apoptosis was observed in $\mathbf{2 8 . 1 \%}$ of TCC cases. The mean AI of all cases was Keywords: Transitional cell carcinoma of bladder, apoptosis, bcl-2, p53

\section{Introduction}

Apoptosis, defined as programmed cell death, plays an important role in tumor growth and development. ${ }^{1}$ Depending on the triggering factor and cell type, there are multiple signaling pathways that lead to activation of the apoptotic machinery. ${ }^{2}$ The bcl-2 family, a group of closely related proteins plays a major role in apoptosis and bcl-2, a member of this family is a potent inhibitor of apoptosis. ${ }^{3}$ P53, a tumor suppressor gene product also plays a role in apoptosis of DNA-damaged cells. ${ }^{4}$ P53 is involved in cell regulation suggesting, that the process of cell proliferation and apoptosis are closely related. ${ }^{4-5}$

In malignancies, alteration in the tumor suppressor gene or in proto-oncogenes may confer a growth advantage to

Received: March 31, 2004; accepted: Aug 12, 2004

Correspondence: Zahra AMIRGHOFRAN, Immunology Department, Medical School, Shiraz University of Medical Science, Shiraz, Iran. Fax: 98-711-2304069, 2359847, e-mail: amirghz@sums.ac.ir
13.7 \pm 24 . No correlation was found between apoptosis and differentiation status of carcinoma. Bcl-2 expression was weakly detected in only one sample. P53 expression was detected in 26 of cases with mean staining index of $102 \pm 96$. A significant correlation between p53 and Ki-67 staining indices was observed $(r=0.521, p=0.001)$. Both p53 and Ki-67 expression showed a good association with the pathological grade $(p=0.0001$ and $p=0.004$, respectively). None of the markers showed significant correlation with AI and no correlation was found between the ratio of AI to PI and other parameters either. In conclusion, the frequency of apoptosis in TCC of bladder appears not to be associated with tumor grade, and with bcl-2, p53 and Ki-67 expression. (Pathology Oncology Research Vol 10, No 3, 154-159) the tumor cells by stimulating cell proliferation. ${ }^{6}$ In recent years, overexpression of $\mathrm{p} 53$ and bcl-2 proteins has been investigated in various malignancies including transitional cell carcinoma of bladder (TCC), and in several studies the prognostic role of these markers, particularly of p53 for TCC has been reported, ${ }^{7-10}$ however, to our knowledge no study on both of these markers in combination with study of apoptosis and cell proliferation indices has been reported. The relationship between tumor expression of p53 and bcl-2 molecules jointly with detection of apoptosis and proliferation may be useful in prognosis of TCC. Using bladder tumor biopsies, the aim of this study was to assess this issue.

\section{Materials and Methods}

Specimens

A total of 49 untreated patients histopathologically proven to have TCC were selected from pathology archives of Shiraz University affiliated hospitals between 
2000-2003. The best paraffin-embedded block of neoplastic tissue was processed for further studies. The neoplastic tissues were either from tumor biopsies, transurethral resection of bladder, or cystectomy specimens. Paraffin blocks were cut into $5 \mu \mathrm{m}$ sections and stained with hematoxylin and eosin. Samples were graded according to ASH classification. ${ }^{11}$ The average patient age was $61 \pm 13$ years (range, $30-65$ years). There were $79 \%$ males versus $21 \%$ female patients.

\section{Immunohistochemistry}

The expression of markers was detected using the threestep streptavidin-biotin immunoperoxidase method. p53 expression was performed using DO1 monoclonal antibody kindly provided by Dr. Nouri AME, from The Royal London Hospital, London. The mouse monoclonal antibody bcl-2 (1:200, Dako, Denmark) was used to detect bcl-2 overexpression. Bcl-2 staining of lymphocytes was used as internal positive control. The polyclonal antibody to Ki-67 (Dako) was used to identify proliferative activity. Briefly, after tissue sections were deparaffinized and rehydrated, they were incubated for 20 minutes with $3 \% \mathrm{H}_{2} \mathrm{O}_{2}$ to inactivate endogenous peroxidase activity. Slides were then heated in $10 \mathrm{mmol} / \mathrm{L}$ citrate buffer, $\mathrm{pH} 6.0$ for 15 minutes to enhance antigen retrieval. Following a $20 \mathrm{~min}-$ utes blocking step with $10 \%$ normal horse serum diluted in phosphate-buffered saline (PBS), the primary antibodies were applied and incubated overnight in a moisture chamber at $4^{\circ} \mathrm{C}$. After treating with biotinylated anti-mouse $\mathrm{IgG}$ and avidin-peroxidase for 30 minutes each, diaminobenzidine $1 \mathrm{mg} / \mathrm{ml}$ in PBS containing $0.03 \%$ hydrogen peroxide was applied as the chromogen. Sections were counterstained with Mayer's hematoxylin for 15 seconds. For each antibody, negative control studies were performed in which PBS was used instead of primary antibody. Paraffin sections from human tonsils and breast cancer with known immunoreactivity to bcl-2, p53 and Ki-67 antigens respectively were used as positive control. To determine the expression of markers, at least 1000 tumor cells were assessed and the proportion of cells showing reactivity was determined. The Ki-67 proliferating index (PI) was defined as the percentage of positive carcinoma cells. Immunostaining intensity was also rated as follows: 0, none; 0.5 , very week; 1 , weak; 2 , moderate and 3 , strong. An index of staining for bcl-2 and p53 expression was determined by multiplying the percentage and intensity of positive tumor cells.

\section{Apoptosis detection}

Apoptosis in 32 of cases were evaluated using TdTmediated dUTP-biotin nick end labeling kit (Boehringer Mannheim, Germany) as recommended by the manufac- turer. Briefly, sections were deparaffinized and rehydrated, and the endogenous peroxidase activity was blocked. The sections were incubated in proteinase $\mathrm{K}$ and then incubated with TUNEL reaction mixture. Slides were incubated with converter-peroxidase solution for a further incubation. The reaction was visualized with diaminobenzidine $/ \mathrm{H}_{2} \mathrm{O}_{2}$. Slides were subsequently washed, counterstained with hematoxylin and mounted. Negative control sections included the above process except the enzyme solution and positive control were prepared by treating sections with $1 \mu \mathrm{g} / \mathrm{ml}$ DNase 10 minutes before the above protocol. Apoptotic cells were identified using light microscope, and a total of 1000 carcinoma cells were evaluated, whereby the apoptotic index (AI) was determined.

\section{Statistics}

All statistical analyses were performed using SPSS software. Mann-Whitney test and Pearson correlation assessed the correlation between expression of markers and the age and sex of patients. Spearman correlation was used to evaluate the association of markers with each other and with grade.

\section{Results}

Tumor specimens archived in paraffin blocks from patients with TCC were examined for the expression of different markers as well as for the presence of apoptotic cells using the TUNEL technique. $6.1 \%$ of cases were grade I, $32.6 \%$ grade II, $26.9 \%$ grade III and $34.7 \%$ were grade IV.

\section{P53 expression}

26 cases showed immunoreactivity for p53 (10-100\% of tumor cells) (Figure 1a). The p53 staining index in the tumors varied from 10 to 300 . The mean value of $\mathrm{p} 53$ index was $109 \pm 96$. Statistical analysis of the relationship between p53 expression and grade of tumors showed a significant correlation $(\mathrm{r}=0.658, \mathrm{p}=0.0001)$, as the mean staining index of p53 expression for low grade (I\&II) patients was $21 \pm 60$ versus $112 \pm 99$ for high grade (III \& IV) ones (Table 1). Determination of the nuclear p53 staining showed $81.5 \%$ p53 positivity in cases with $\geq 5 \%$ tumor cell staining for Ki-67, showing a good correlation between the expression of these two markers ( $\mathrm{r}=0.521$, $\mathrm{p}=0.001$ ) (Figure 2).

\section{Ki-67 expression}

Most (39 cases) of TCC cases showed Ki-67 positivity of more than $5 \%$. The mean Ki-67 proliferating index was $24 \pm 22$. A significant correlation between Ki-67 expression 
and pathological grade was observed $(\mathrm{r}=0.441, \mathrm{p}=0.004)$, showing an increase in the expression of Ki-67 in less differentiated tumor cells.

\section{Bcl-2 expression}

Bcl-2 staining was observed in nearly none of the cases examined (Figure $1 b$ ). Only in one specimen with grade II a $5 \%$ weakly staining was detected.

\section{Apoptotic index}

TdT-mediated nick end labeling method of determining apoptosis in the TCC samples was positive in 9 cases (28.1\%) (Figure 1c). A mean AI of $13.7 \pm 24$ for all samples was obtained. AI in different grades of carcinoma is shown in Table 1. No correlation between AI and differentiation status of carcinoma as well as markers examined was obtained. The ratio of apoptotic cells to Ki-67 positive cells (AI/PI) was determined. This ratio showed a significant correlation with AI $(r=0.994, p=0.0001)$ but not with other parameters.

No correlation between sex and age of patients with grade, AI and other parameters was obtained.

\section{Discussion}

Apoptosis is a distinct mode of cell death that is responsible for deletion of cells in malignant tumors as well as normal tissues. ${ }^{12}$ The rate of apoptosis in malignant tumors is an important factor for tumor growth. ${ }^{1-2}$ Few studies have been done on the rate of apoptosis in TCC. In the present work, apoptosis was detected in nearly $28 \%$ of patients with TCC of bladder. No correlation between AI and grade of tumors was observed, indicating that this index may not be valuable as a prognostic marker. Similar results have been reported by King et al. on 45 patients with no significant correlation between the frequency of apoptosis and the pathological grade and stage of the tumors. ${ }^{13}$ In a search through Medline a report for apoptosis detected by a monoclonal antibody recognizing single-stranded DNA regions was also found in that all 103 cases studied were positive and AI tended to increase with increasing grade. ${ }^{14}$ Apoptotic cells with mean percentage of $22 \pm 13$ in TCC of bladder was reported by Roman et al. ${ }^{15}$ In two other studies the frequency of apoptosis using TUNEL technique in TCC of the renal pelvis and ureter has been studied, ${ }^{16,17}$ and a close association of the incidence of apoptosis with the tumor grade of TCC was observed, indicating that apoptotic cell death may play a role in the tumor progression of renal pelvic and ureteral TCC.

The correlation between apoptosis and proliferation or Ki-67 index of tumor tissues is of importance because the rate of tumor cell accumulation is not only dependent on the rate of cell death but also on the rate of tumor cell proliferation. ${ }^{1}$ Decrease in apoptosis/proliferation rate may confer a growth advantage to the tumor cells. ${ }^{12} \mathrm{AI}$ in our TCC cases was not correlated with Ki-67 staining index, and after determination the apoptotic/Ki-67 indices rate we found no difference between patients with dissimilar pathological grade, indicating the minor role of apoptosis rate in the growth of transitional tumor cells.
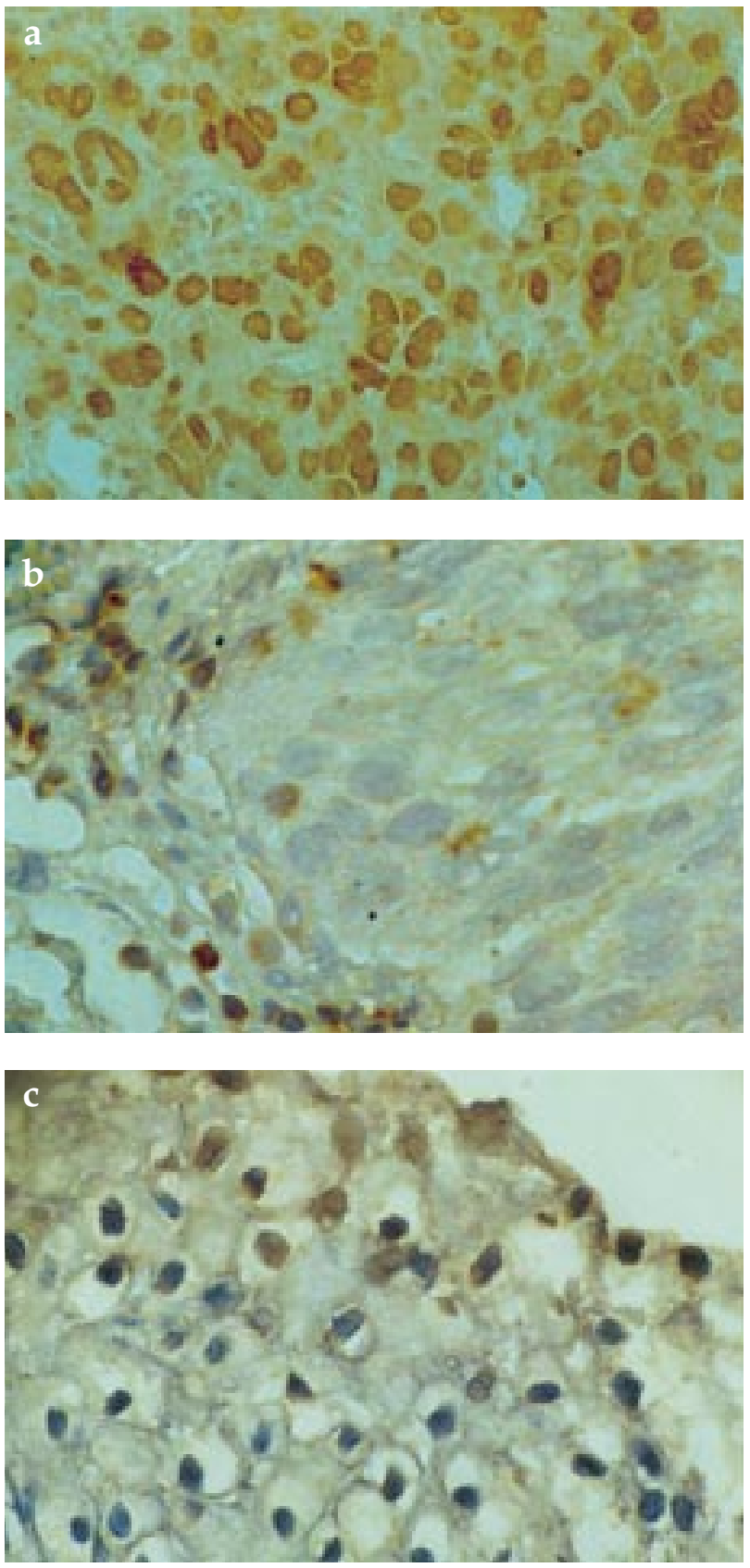

Figure 1. (a) Nuclear immunostaining of p53 in tumor cells of TCC (X400). (b) Bcl-2 negative staining of tumor cells. Lymphocytes are positive (X400) (c) Positive TUNEL stain observed in tumor cells nuclei. (X320). 
Table 1. Comparison between different grades of tumors and the corresponding staining indices, proliferation index (PI) and apoptotic index (AI) in TCC patients.

\begin{tabular}{lcccrr}
\hline Grade & Bcl-2 staining index & P53 staining index & Ki-67 index(PI) & AI & AI/PI \\
\hline II & $0.4 \pm 1.44$ & $24.3 \pm 64$ & $12.42 \pm 11$ & $11 \pm 23$ & $2.8 \pm 6.1$ \\
III & 0.0 & $54.7 \pm 63$ & $33.9 \pm 28$ & $11.8 \pm 24$ & $0.05 \pm 0.1$ \\
IV & 0.0 & $145 \pm 107$ & $32.7 \pm 22$ & $23.5 \pm 17$ & $0.6 \pm 0.9$ \\
& $\mathrm{P}=0.4$ & $\mathrm{P}=0.0001$ & $\mathrm{P}=0.004$ & $\mathrm{P}=0.4$ & $\mathrm{P}=0.3$ \\
\hline
\end{tabular}

Apoptosis is modulated by the products of a large number of evolutionary conserved oncogenes and tumor suppressor genes. ${ }^{18,19} \mathrm{Bcl}-2$ is considered a downstream regulator of apoptosis because it inhibits apoptosis by a variety of stimuli ${ }^{3}$. Bcl-2 staining was nearly negative in our study. In fact, loss of bcl-2 is expected to be associated with higher rate of apoptosis, resulting in a decrease of tumor growth. However, no correlation was found between bcl-2 expression and apoptosis in our cases, suggesting that the expression level of bcl-2 protein may not be the critical factor that determines the degree of apoptosis, and other factors may be involved.

The protein $\mathrm{p} 53$, among its many diverse functions acts as a factor that up-regulates the activity of several genes that modulate apoptosis. ${ }^{20}$ The mutant p53, however, functions as a substitute for bcl-2 and inhibits apoptosis. ${ }^{21}$ In previous studies the role of p53 in TCC of bladder has been well studied and overexpression correlated with grade, stage, progression and survival. ${ }^{710}$ Concordantly, we showed that $\mathrm{p} 53$ positivity is strongly associated with high histological grade, indicating the important role of this protein in TCC prognosis. P53 expression was correlated with proliferation index determined by Ki-67 staining as well, showing that p53 immunopositivity is associated with active cell division. The diverse effect of p53 and bcl-2 on cell death has encouraged investigators to search for a possible reciprocal relationship between expression as well as prognostic significance of these two markers in various malignancies

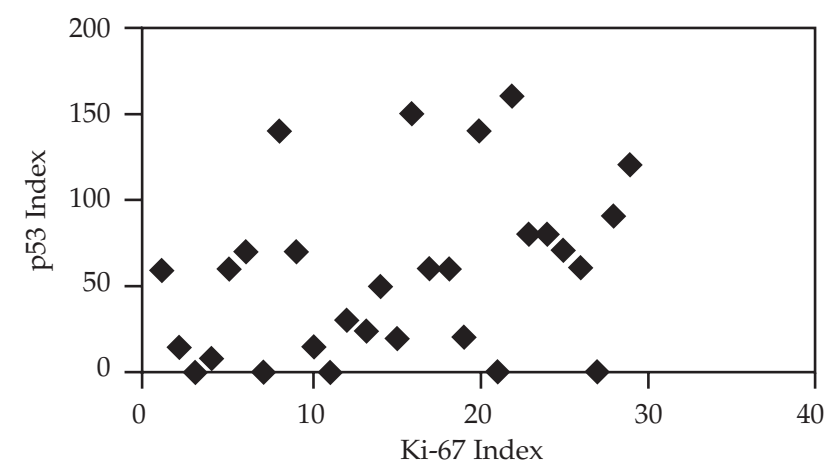

Figure 2. Relationship between $p 53$ and Ki-67 indices in TCC patients $(P=0.001)$.

Vol 10, No 3, 2004 including TCC of bladder. In a study on 119 patients $61 \%$ and $17 \%$ of cases were positive for expression of $\mathrm{p} 53$ and bcl-2, respectively. P53 expression was correlated with stage but not bcl-2 with any clinicopathologic parameters. However, co-expression of p53 and bcl-2 was associated with unfavorable prognosis. ${ }^{22}$ In another study bcl2 expression was studied in 83 patients with invasive TCC treated with radiotherapy, and a poor outcome in patients expressing bcl-2 and p53 was found. ${ }^{23}$ Cytoplasmic expression of bcl-2 was also observed in $54 \%$ and p53 in $37 \%$ of TCC patients less than 40 years old, and was not associated with tumor progression. ${ }^{24}$ In our study bcl-2, in contrast to p53. showed no correlation with grade of tumor or with apoptosis, and there was no relationship between bcl-2 and p53 expression. This result may be in part due to loss of bcl-2 expression observed in our cases. This finding that bcl-2 positivity is rare in TCC of bladder is almost consistent with two other reports by Kirsh et al. and King et al. in which only $7.0 \%$ and less than $5.3 \%$ of TCC samples demonstrated bcl-2 overexpression, respectively. ${ }^{13,25}$ Infrequent expression of bcl-2 noted in these studies raises the need for more investigations regarding the role of genetic background in bladder tumorigenesis as well as the role of other markers involved in the regulation of apoptosis in TCC. It has been reported that in superficial bladder cancers, p53 mutations may inactivate the transcription of bax gene. ${ }^{26}$ Bax is a member of the bcl-2 family and has a pro-apoptotic effect. ${ }^{27}$ Although the phenomenon of p53 overexpression in TCC has mostly been reported to have underlying genetic mutations, ${ }^{28}$ the authors can not conclude that the p53 positivity observed in our cases are accompanied by p53 mutation, and hence the exact role of mutant and/or wild-type p53 expression on frequency of apoptosis and bcl-2 in TCC requires more studies.

In conclusion, this study showed that, in TCC of bladder, the degree of apoptosis is not correlated with the cellular proliferation and p53 and bcl-2 expression, and that there appeared to be rare positive staining for bcl-2.

\section{Acknowledgement}

This work was supported by grants 78-696 and 80-1364 from Shiraz University of Medical Sciences. 


\section{References}

1. Hickman JA. Apoptosis and tumorigenesis. Curr Opin Genet Dev 12:67-72, 2002

2. Rossi D, Gaidano $G$. Messengers of cell death: apoptotic signaling in health and disease. Haematologica 88:212-218, 2003

3. Burlacu A. Regulation of apoptosis by $\mathrm{Bcl}-2$ family proteins. $\mathrm{J}$ Cell Mol Med 7:249-257, 2003

4. Batinac T, Gruber F, Lipozencic J et al. Protein p53-structure, function, and possible therapeutic implications. Acta Dermatovenerol Croat 11:225-230, 2003

5. Laiho $M$, Latonen $L$. Cell cycle control, DNA damage checkpoints and cancer. Ann Med 35:391-397, 2003

6. Sherr CJ. Principles of tumor suppression. Cell 23; 116:235246, 2004

7. Rodriguez-Alonso A, Pita-Fernandez S, Gonzalez-Carrero J, Nogueira-March JL: Multivariate analysis of survival, recurrence, progression and development of metastasis in $\mathrm{T} 1$ and T2a transitional cell bladder carcinoma. Cancer 15; 94:16771684, 2002

8. Lin Z, Kim H, Park H, et al. The expression of bcl-2 and bcl6 protein in normal and malignant transitional epithelium. Urol Res 31:272-275, 2003

9. Yu DS, Chang SY. The expression of oncoproteins in transitional cell carcinoma: its correlation with pathological behavior, cell cycle and drug resistance. Urol Int 69:46-50, 2002

10. Llopis J, Alcaraz A, Ribal MJ, et al. p53 expression predicts progression and poor survival in T1 bladder tumors. Eur Urol 37:644-653, 2000

11. World Health Organization. Histological typing of urinary bladder tumors. 10, 1973

12. Budd RC. Activation-induced cell death. Curr Opin Immunol 13:356-362, 2001

13. King ED, Matteson J, Jacobs SC, Kyprianou N. Incidence of apoptosis, cell proliferation and bcl-2 expression in transitional cell carcinoma of the bladder: association with tumor progression. J Urol 155:316-320, 1996

14. Korkolopoulou P, Konstantinidou AE, Christodoulou P, et al. Apoptosis in bladder carcinomas detected with monoclonal antibody to single-stranded DNA: relation to cell cycle regulators and survival. Urology 1; 56:516-520, 2000
15. Roman S, Petrusca D, Moldovan I, et al. Evaluation of apoptosis of tumor and of apparently normal cells in human renal carcinoma. Immunol Lett 15; 67:15-22, 1999

16. Kong C, Zhang X, Takenaka I. Apoptotic cell death and Smad4 expression in transitional cell carcinoma of the renal pelvis and ureter. Int J Urol 8:386-390, 2001

17. Zhang X, Kong C, Takenaka I. Evaluation of cell proliferation, apoptosis, and angiogenesis in transitional cell carcinoma of the renal pelvis and ureter. Urology 57:981-985, 2001

18. Townson JL, Naumov GN, Chambers AF. The role of apoptosis in tumor progression and metastasis. Curr Mol Med 3:631642,2003

19. Vermeulen K, Berneman ZN, Van Bockstaele DR. Cell cycle and apoptosis. Cell Prolif 36165-36175, 2003

20. Bargonetti J, Manfredi JJ. Multiple roles of the tumor suppressor p53. Curr Opin Oncol 14:86-91, 2002

21. Lu QL, Abel P, Foster CS, Lalani EN. bcl-2: role in epithelial differentiation and oncogenesis. Hum Pathol 27:102-110, 1996

22. Uchida T, Minei S, Gao JP, et al. Clinical significance of p53, MDM2 and bcl-2 expression in transitional cell carcinoma of the bladder. Oncol Rep 9:253-9, 2002

23. Ong F, Moonen LM, Gallee MP, et al. Prognostic factors in transitional cell cancer of the bladder: an emerging role for Bcl-2 and p53. Radiother Oncol 61:169-175, 2001

24. Asci R, Yildiz L, Sarikaya S, et al. p53 and bcl-2 overexpression as associated risk factors in patients 40 years old or less with transitional cell carcinoma of the bladder. Urol Int 67:3440, 2001

25. Kirsh EJ, Baunoch DA, Stadler WM. Expression of bcl-2 and bcl-X in bladder cancer. J Urol 159:1348-1353, 1998

26. Pfister $C$, Flaman JM, Dunet F, et al. p53 mutations in bladder tumors inactivate the transactivation of the p21 and Bax genes, and have a predictive value for the clinical outcome after bacillus Calmette-Guerin therapy. J Urol 162:69-73, 1999

27. Harada H, Grant $S$. Apoptosis regulators. Rev Clin Exp Hematol 7:117-138, 2003

28. Smith ND, Rubenstein JN, Eggener SE, Kozlowski JM. The p53 tumor suppressor gene and nuclear protein: basic science review and relevance in the management of bladder cancer. $\mathrm{J}$ Urol 169: 1219-1228, 2003 